\title{
In Silico Populations Optimized on Optogenetic Recordings Predict Drug Effects in Human Induced Pluripotent Stem Cell-Derived Cardiomyocytes
}

\author{
Michelangelo Paci ${ }^{1}$, Elisa Passini ${ }^{2}$, Aleksandra Klimas ${ }^{3}$, Stefano Severi ${ }^{4}$, Jari Hyttinen ${ }^{1}$, Blanca \\ Rodriguez ${ }^{2}$, Emilia Entcheva ${ }^{3}$ \\ ${ }^{1}$ Faculty of Biomedical Sciences and Engineering, Tampere University of Technology, Tampere, \\ Finland \\ ${ }^{2}$ Department of Computer Science, University of Oxford, Oxford, United Kingdom \\ ${ }^{3}$ Department of Biomedical Engineering, The George Washington University, Washington DC, \\ United States of America \\ ${ }^{4}$ Department of Electrical, Electronic and Information Engineering, University of Bologna, Cesena, \\ Italy
}

\begin{abstract}
All-optical high-throughput systems allow simultaneous high resolution action potential $(A P)$ and $\mathrm{Ca}^{2+}$ transient (CaTr) measurements from cardiomyocytes within multicellular context, offering means to speed up in vitro drug tests. Here, we aim to develop experimentallyconstrained in silico models of human induced pluripotent stem cell-derived cardiomyocytes (hiPSC-CMs) and hiPSC-CM populations to predict drug effects in humans, by leveraging functional data obtained by all-optical means. Using multi-objective genetic algorithms (MoGAs), we constructed three control populations of in silico hiPSC-CMs, constrained with experimental data of APs and CaTrs recorded at room temperature and non-paced conditions from three different plates containing hiPSCCM syncytia. We then simulated the effect of increasing doses of Diltiazem (130 models), Cisapride (200 models) and Astemizole (200 models) in the three populations, respectively. Comparing model predictions with the experimental drug administration (not used for the optimization/calibration of the populations) revealed good agreement with experiments: e.g. Diltiazem shortened APs while Astemizole and Cisapride prolonged APs.
\end{abstract}

\section{Introduction}

Optical approaches offer contact-free high-resolution measurements of key electromechanical parameters in cardiomyocytes, e.g. action potentials (AP), $\mathrm{Ca}^{2+}$ transients (CaTr), or contraction. Recently, all-optical highthroughput systems allowed simultaneous AP and $\mathrm{CaTr}$ measurements from cardiomyocytes within multicellular context, offering means to speed up in vitro drug tests
[1,2]. In this work, we aim (i) to calibrate/optimize populations of in silico models of human induced pluripotent stem cell-derived cardiomyocytes (hiPSCCMs) by means of simultaneous optically recorded data of APs and CaTrs in control conditions and (ii) to assess the predictive power of our in silico populations during the administration of three specific drugs (Diltiazem, Cisapride and Astemizole) plus a fourth drug (Dofetilide) as positive control.

\section{Methods}

\subsection{Experimental dataset}

The experimental dataset consists in APs and CaTrs optically recorded from hiPSC-CMs syncytia (CDI iCell ${ }^{2}$ cardiomyocytes) at room temperature under non-paced conditions. Recordings were performed in control conditions and after administration of one of the following drugs: Diltiazem (mainly an $\mathrm{I}_{\mathrm{CaL}}$ blocker), Cisapride and Astemizole (both mainly $\mathrm{I}_{\mathrm{Kr}}$ blockers). In detail, recordings were performed on three plates (384-well format), where $\mathrm{APs}$ and CaTrs were recorded in negative control (administration of $0.1 \%$ DMSO, C-) and then administered with four increasing doses (D1, D2, D3 and D4) of Diltiazem (Plate 1), Cisapride (Plate 2) and Astemizole (Plate 3). Furthermore, $0.5 \mathrm{nM}$ Dofetilide was tested on all the plates as positive control $(\mathrm{C}+)$. APs and CaTrs were recorded from five $\mathrm{C}$ - samples and from six samples each of C+, D1, D2, D3 and D4. Experimental biomarkers for the three plates are reported in Table 1. For each biomarker, we had five measurements in $\mathrm{C}$ - and six measurements in the other conditions. Biomarkers are: AP and $\mathrm{CaTr}$ cycle length (Vm CL and $\mathrm{Ca} C \mathrm{CL})$, duration at $30 \%, 50 \%$ and $90 \%$ of $\mathrm{AP}\left(\mathrm{APD}_{30}, \mathrm{APD}_{50}\right.$ and $\left.\mathrm{APD}_{90}\right)$ and 
Table 1. Lower bounds (LB) and upper bounds (UB) for the five AP biomarkers and the six CaTr biomarkers for the three plates in $\mathrm{C}$ - conditions. Values in bold were computed as mean $\pm 2 \mathrm{SD}$, values in italic were computed as mean $\pm 3 \mathrm{SD}$, to be used in section 2.2.2.

\begin{tabular}{|c|c|c|c|c|c|c|}
\hline & \multicolumn{2}{|c|}{ Plate 1} & \multicolumn{2}{|c|}{ Plate 2} & \multicolumn{2}{|c|}{ Plate 3} \\
\hline & LB & UB & LB & UB & LB & UB \\
\hline \multirow{2}{*}{ Vm CL (ms) } & 4989 & 8423 & 2172 & 4255 & 3079 & 5222 \\
\hline & 4690 & 9019 & 1842 & 4712 & 3003 & 5461 \\
\hline \multirow{2}{*}{$\mathrm{APD}_{90}(\mathrm{~ms})$} & 786 & 1145 & 938 & 1116 & 831 & 1275 \\
\hline & 756 & 1196 & 916 & 1142 & 721 & 1386 \\
\hline \multirow{2}{*}{$\mathrm{APD}_{50}(\mathrm{~ms})$} & 520 & 752 & 736 & 910 & 698 & 921 \\
\hline & 504 & 772 & 717 & 934 & 672 & 958 \\
\hline \multirow{2}{*}{$\mathrm{APD}_{30}(\mathrm{~ms})$} & 391 & 575 & 515 & 673 & 459 & 719 \\
\hline & 377 & 586 & 502 & 693 & 438 & 767 \\
\hline \multirow{2}{*}{$\begin{array}{l}\text { Vm Tri90-30 } \\
(\mathrm{ms})\end{array}$} & 393 & 571 & 418 & 478 & 253 & 564 \\
\hline & 369 & 611 & 406 & 491 & 175 & 641 \\
\hline \multirow{2}{*}{$\mathrm{Ca}$ CL (ms) } & 4997 & 8429 & 2174 & 4255 & 3084 & 5218 \\
\hline & 4701 & 9028 & 1845 & 4711 & 3019 & 5455 \\
\hline \multirow{2}{*}{$\mathrm{CTD}_{90}(\mathrm{~ms})$} & 904 & 2306 & 1564 & 2023 & 1434 & 2322 \\
\hline & 554 & 2656 & 1541 & 2093 & 1310 & 2460 \\
\hline \multirow{2}{*}{$\mathrm{CTD}_{50}(\mathrm{~ms})$} & 804 & 1163 & 979 & 1095 & 918 & 1149 \\
\hline & 751 & 1194 & 972 & 1106 & 882 & 1178 \\
\hline \multirow{2}{*}{$\mathrm{CTD}_{30}(\mathrm{~ms})$} & 644 & 903 & 828 & 903 & 761 & 931 \\
\hline & 615 & 918 & 825 & 909 & 734 & 945 \\
\hline \multirow{2}{*}{$\begin{array}{l}\text { Ca tRise } \\
(\mathrm{ms})\end{array}$} & 221 & 373 & 331 & 413 & 286 & 443 \\
\hline & 196 & 382 & 312 & 423 & 277 & 463 \\
\hline \multirow{2}{*}{$\begin{array}{l}\text { Ca Tri90-30 } \\
(\mathrm{ms})\end{array}$} & 258 & 1545 & 727 & 1121 & 667 & 1424 \\
\hline & 0 & 1867 & 681 & 1186 & 567 & 1545 \\
\hline
\end{tabular}

of $\mathrm{CaTr}\left(\mathrm{CTD}_{30}, \mathrm{CTD}_{50}, \mathrm{CTD}_{90}\right), \quad \mathrm{AP}$ and $\mathrm{CaTr}$ triangulation (Vm Tri90-30=APD $90-\mathrm{APD}_{30}$ and $\mathrm{Ca}$ Tri90$\left.30=\mathrm{CTD}_{90}-\mathrm{CTD}_{30}\right)$ and $\mathrm{CaTr}$ time to rise from $10 \%$ to $90 \%$ (Ca tRise). For each plate and biomarker, we considered as lower bounds $\left(\mathrm{LB}_{\mathrm{i}}, \mathrm{LB}_{3 \mathrm{SD}, \mathrm{i}}\right)$ the smallest of mean-2SD and mean-3SD, while as upper bounds $\left(\mathrm{UB}_{\mathrm{i}}, \mathrm{UB}_{3 \mathrm{SD}, \mathrm{i}}\right)$ the maximum of mean+2SDand mean+3SD of the five control measurements for the biomarker i (see Table 1, values in bold for $\pm 2 \mathrm{SD}$ and in italic for $\pm 3 \mathrm{SD}$ ).

\subsection{In silico modeling}

\subsubsection{Adapting the hiPSC-CM model to room temperature}

Simulations were performed with the recently published Paci2018 hiPSC-CM model [3], which was tuned and validated to simulate APs and CaTrs at $37^{\circ} \mathrm{C}$. To simulate the aforementioned experiments, we adapted the model to room temperature $\left(21^{\circ} \mathrm{C}\right)$ by rescaling the model time constants for the main ionic currents according to the Q10 factors reported in Table 2 .
Table 2. Q10 factors to translate the model from $37^{\circ} \mathrm{C}$ to $21^{\circ} \mathrm{C}$ [4-7].

\begin{tabular}{ll}
\hline Ionic current & Q10 factors \\
\hline $\mathrm{I}_{\mathrm{Na}}$ & 2.00 \\
$\mathrm{I}_{\mathrm{NaL}}$ & 2.20 \\
$\mathrm{I}_{\mathrm{CaL}}$ & 2.10 \\
$\mathrm{I}_{\mathrm{f}}$ & 4.50 \\
$\mathrm{I}_{\mathrm{Kr}}$ activation & 4.55 \\
$\mathrm{I}_{\mathrm{Kr}}$ inactivation & 3.08 \\
$\mathrm{I}_{\mathrm{Ks}}$ & 2.00 \\
$\mathrm{I}_{\text {to }}$ & 2.00 \\
\hline
\end{tabular}

\subsubsection{Multi-objective genetic algorithms}

Instead of a canonical approach to develop a population of in silico models, as in [8,9], here we opted for multiobjective genetic algorithms (MoGAs) [10]: they allow the concurrent optimization of many fitness functions, to find an optimal population. We chose to sample the following 22 parameters: (i) the maximum conductances/currents of $\mathrm{I}_{\mathrm{Na}}, \mathrm{I}_{\mathrm{f}}, \mathrm{I}_{\mathrm{CaL}}, \mathrm{I}_{\mathrm{to}}, \mathrm{I}_{\mathrm{Ks}}, \mathrm{I}_{\mathrm{Kr}}, \mathrm{I}_{\mathrm{K} 1}, \mathrm{I}_{\mathrm{NCX}}, \mathrm{I}_{\mathrm{NaK}}, \mathrm{I}_{\mathrm{pCa}}, \mathrm{I}_{\mathrm{NaL}}, \mathrm{I}_{\mathrm{RyR}}, \mathrm{I}_{\mathrm{SERCA}}$; (ii) activation and inactivation time constants of $\mathrm{I}_{\mathrm{Na}}, \mathrm{I}_{\mathrm{CaL}}$ and $\mathrm{I}_{\mathrm{RyR}}$; (iii) adaptation time constant and half inactivation $\mathrm{Ca}^{2+}$ concentration of $\mathrm{I}_{\mathrm{RyR}}$; (iv) $\mathrm{I}_{\mathrm{SERCA}}$ half saturation constant. We defined two fitness functions, based on AP and CaTr biomarkers, respectively.

$$
\begin{gathered}
E r r=\sum_{1}^{\text {Nbiomarkers }} \operatorname{err}_{i} \\
\operatorname{err}_{i}=\frac{\left(b_{i, s i m}<L B_{i}\right)\left(b_{i, s i m}-L B_{i}\right)^{2}+\left(b_{i, s i m}>U B_{i}\right)\left(b_{i, s i m}-U B_{i}\right)^{2}}{0.5\left(L B_{i}+U B_{i}\right)},
\end{gathered}
$$

where $b_{i, s i m}$ is the $i$-th simulated biomarker, $L_{B}$ the $i$-th experimental lower bound for $b_{i, s i m}, U B_{i}$ the $i$-th experimental upper bound for $b_{i, s i m}$ and Nbiomarkers the number of biomarkers computed on the experimental APs ( 5 biomarkers) and CaTrs (6 biomarkers). Shortly, if the simulated $\mathrm{i}$-th biomarker is smaller than $\mathrm{LB}_{\mathrm{i}}$ or greater than $\mathrm{UB}_{\mathrm{i}}$, the error is computed as the squared distance between the simulated biomarker and the bound, normalized by the center of mass of $\left[\mathrm{LB}_{\mathrm{i}}, \mathrm{UB}_{\mathrm{i}}\right]$. The biomarkers used to constrain the fitness functions are listed in Section 2.1 and Table 1. MoGAs parameters were: maximum number of models $=200$ and maximum number of generations $=40$. For each of the 200 parameter sets optimized by MoGAs, we then run the simulation up to steady state (500s) and rechecked that all the biomarkers were included in the ranges $\left[\mathrm{LB}_{3 \mathrm{SD}, \mathrm{i}}, \mathrm{UB}_{3 \mathrm{SD}, \mathrm{i}}\right]$, to include in the population also those parameter sets at the very edge of the ranges $\left[\mathrm{LB}_{\mathrm{i}}, \mathrm{UB}_{\mathrm{i}}\right]$.

\subsubsection{Drug tests}

To assess the predictive power of our three populations of hiPSC-CM models, we then simulated Diltiazem, Cisapride, Astemizole and Dofetile $(\mathrm{C}+)$ at the four doses 
Table 3. $\mathrm{IC}_{50}$ and Hill's coefficients (in brackets) for the four drugs in rows 2-7 [11]. The experimentally tested drug doses are reported in $\mu \mathrm{M}$ in rows 8-12.

\begin{tabular}{|c|c|c|c|c|c|}
\hline & & Diltiazem & Cisapride & Astemizole & Dofetilide \\
\hline \multirow{6}{*}{ 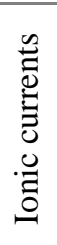 } & $\mathrm{I}_{\mathrm{Na}}$ & 22.4 & 333.7 & 3 & 162.1 \\
\hline & & (1.29) & (1) & (1.95) & (1) \\
\hline & $\mathrm{I}_{\mathrm{Kr}}$ & 13.2 & 0.02 & 0.004 & 0.03 \\
\hline & & (1.16) & (1.04) & $(0.78)$ & (1.2) \\
\hline & $\mathrm{I}_{\mathrm{CaL}}$ & 0.76 & 11.8 & 1.1 & 26.7 \\
\hline & & (1.14) & (1) & (1.66) & (1) \\
\hline \multirow{5}{*}{ 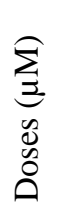 } & $\mathrm{C}+$ & --- & --- & --- & 0.0005 \\
\hline & D1 & 0.01 & 0.0032 & 0.0001 & --- \\
\hline & D2 & 0.1 & 0.01 & 0.001 & --- \\
\hline & D3 & 1 & 0.0316 & 0.01 & --- \\
\hline & D4 & 10 & 0.1 & 0.1 & --- \\
\hline
\end{tabular}

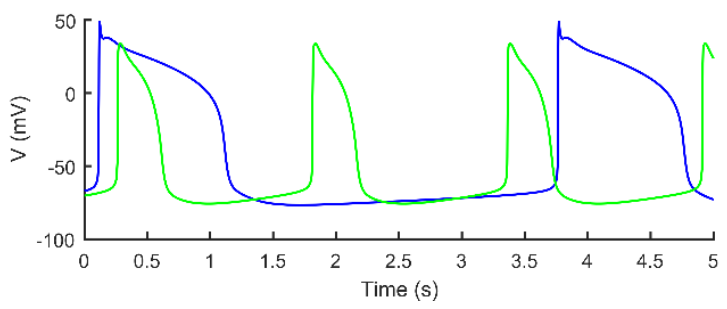

Figure 1. Spontaneous APs simulated by the Paci2018 model at $37^{\circ} \mathrm{C}$ (green) and $21^{\circ} \mathrm{C}$ (blue).

tested experimentally. Of note, the biomarkers computed on APs and CaTrs after drug administration were not used for the parameter set identification with MoGAs. Drug administration was simulated with the single pore block model on the three control populations, as in [8]. In Table 3 we reported the $\mathrm{IC}_{50}$ and the Hill's coefficients for the four drugs, together with the experimentally administered doses and the blocking effects on the ionic currents.

\section{Results}

Figure 1 compares the Paci2018 APs at $37^{\circ} \mathrm{C}$ and $21^{\circ} \mathrm{C}$ : as expected, the rate of spontaneous APs is slower and APD is longer at $21^{\circ} \mathrm{C}$. This step helped also shifting AP and $\mathrm{CaTr}$ biomarkers closer to the experimental values recorded in C- conditions. MoGAs optimization produced three in silico control hiPSC-CMs populations, each representing the variability of a specific experimental plate. We then tested on each of these populations the corresponding drug which was tested experimentally. Specifically, Plate 1 (Diltiazem) contains 130 models, while Plate 2 (Cisapride) and Plate 3 (Astemizole) 200 models. Figure 2, 3 and 4 show the model distributions within the $\left[\mathrm{LB}_{3 \mathrm{SD}}, \mathrm{UB}_{3 \mathrm{SD}}\right]$ variability intervals of the biomarkers in each plate. For each biomarker, simulations and experimental variability ranges are reported in $\mathrm{C}-, \mathrm{C}+$,
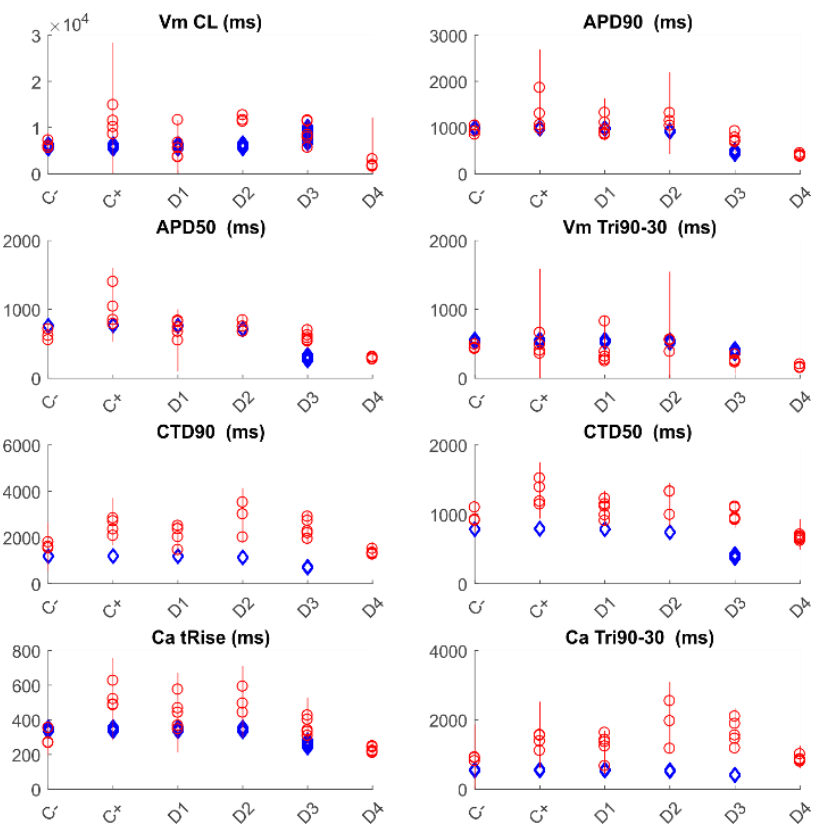

Figure 2. Diltiazem (D1, D2, D3, D4) and Dofetilide (C+) effects on the biomarkers, compared to control (C-). Red circles represent the experimentally recorded biomarkers, and the red bars the experimental variability intervals, for each dose. Blue diamonds are the simulated biomarkers.

D1, D2, D3 and D4 of the plate-specific drugs (Diltiazem was not simulated at D4). The population biomarkers are perfectly included within the experimental variability ranges for C-, thanks to the calibration process. However, drug simulations (D1, D2, D3 and D4) are also in agreement with the drug-induced changes observed in the experiment, although these experiments were not used for the optimization process. For example, Diltiazem shortens $\mathrm{APD}_{90}$ and reduces Vm Tri90-30. Conversely, Cisapride and Astemizole prolong $\mathrm{APD}_{90}$ and $\mathrm{CTD}_{90}$, increase $\mathrm{Ca}$ tRise and the triangulation of both $\mathrm{AP}$ and $\mathrm{CaTr}$.

\section{Conclusions}

In this work we proposed a proof-of-concept optimization of in silico populations by means of MoGAs. We observed in particular that by optimizing an in silico population on the control experiments of different plates, we then obtain qualitative agreement between simulated and experimental drug effects, without using for a following optimization the experimentally recorded data on the same plate after drug administration. However, for some of the biomarkers, e.g. $\mathrm{APD}_{50}$ and $\mathrm{APD}_{30}$, for all the three drugs, especially at the highest drug doses, the simulated drug effects look amplified compared to experiments, possibly due to nonspecific and/or multi-channel drug effects experimentally that are not reflected in the model. This work therefore 

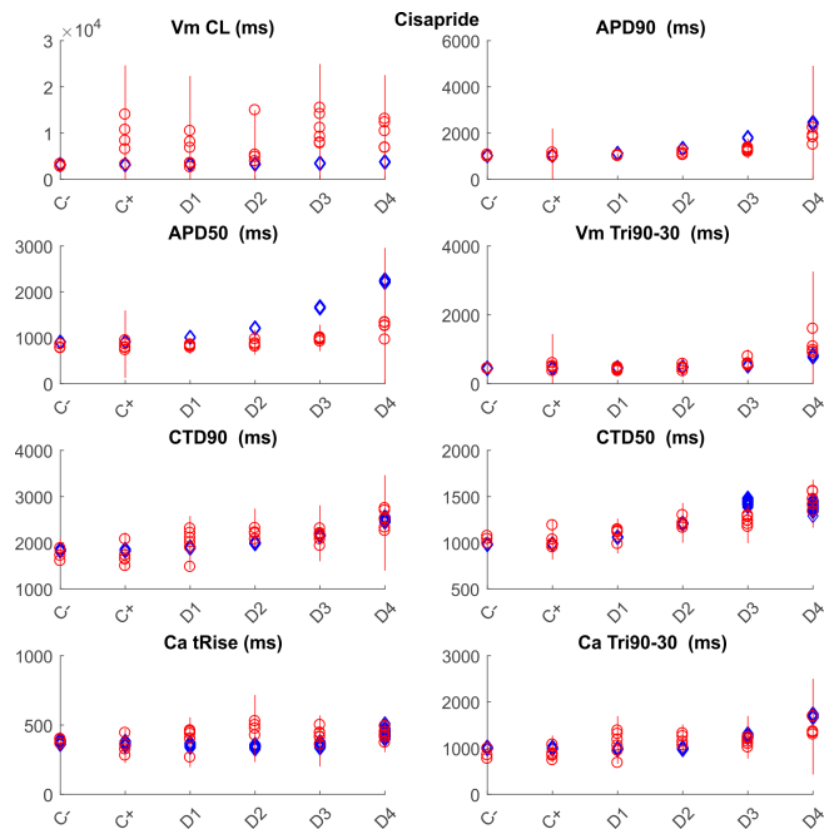

Figure 3. Cisapride (D1, D2, D3, D4) and Dofetilide (C+) effects on the biomarkers, compared to control (C-). Red circles represent the experimentally recorded biomarkers, and the red bars the experimental variability intervals, for each dose. Blue diamonds are the simulated biomarkers.
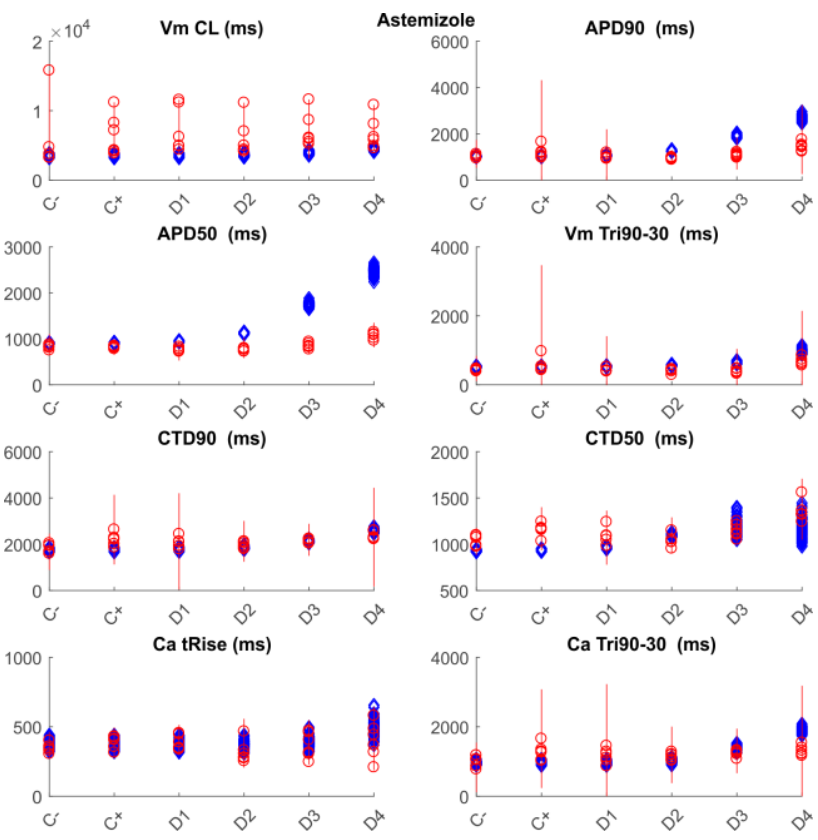

Figure 4. Astemizole (D1, D2, D3, D4) and Dofetilide (C+) effects on the biomarkers, compared to control (C-). Red circles represent the experimentally recorded biomarkers, and the red bars the experimental variability intervals, for each dose. Blue diamonds are the simulated biomarkers. shows that optically-obtained data are suitable for tuning populations of in silico models of hiPSC-CMs and that MoGAs represent an alternative, or can be combined, to canonical approaches for generating populations of in silico models.

\section{Acknowledgements}

Dr. Michelangelo Paci was supported by the Academy of Finland (decision number 307967). The authors also thank CSC - IT Centre for Science (Finland) and ARC Advanced Research Computing of University of Oxford (UK) for generous computational resources.

\section{References}

[1] Klimas A et al. OptoDyCE as an automated system for highthroughput all-optical dynamic cardiac electrophysiology. Nat Commun. 2016;7(May):11542.

[2] Entcheva E et al. All-optical control of cardiac excitation: Combined high-resolution optogenetic actuation and optical mapping. J Physiol. 2016;9(November 2014):2503-10.

[3] Paci M et al. Automatic optimization of an in silico model of human iPSC derived cardiomyocytes recapitulating calcium handling abnormalities. Front Physiol. 2018;9(June):709.

[4] O'Hara T et al. Simulation of the undiseased human cardiac ventricular action potential: model formulation and experimental validation. PLoS Comput Biol. 2011;7(5):e1002061.

[5] ten Tusscher KHWJ et al. A model for human ventricular tissue. Am J Physiol Hear Circ Physiol. 2004;286(4):H15731589.

[6] Stieber J et al. channels: Hyperpolarization-activated, cyclic nucleotide-gated $(\mathrm{HCN})$ channels: from genes to function. In: Cardiac Electrophysiology: From Cell to Bedside. 5th ed. Elsevier; 2009. p. 1184.

[7] Mauerhöfer $M$ et al. Effects of Temperature on Heteromeric Kv11.1a/1b and Kv11.3 Channels. Biophys J. 2016;111(3):504-23.

[8] Paci M et al. Phenotypic variability in LQT3 human induced pluripotent stem cell-derived cardiomyocytes and their response to antiarrhythmic pharmacologic therapy: An in silico approach. Hear Rhythm. 2017;14(11):1704-12.

[9] Paci $M$ et al. A population of in silico models to face the variability of human induced pluripotent stem cell-derived cardiomyocytes: the hERG block case study. In: Computing in Cardiology. 2016. p. 1189-92.

[10]Konak A et al. Multi-objective optimization using genetic algorithms: A tutorial. Reliab Eng Syst Saf. 2006;91(9):9921007.

[11]Crumb WJ et al. An evaluation of 30 clinical drugs against the comprehensive in vitro proarrhythmia assay (CiPA) proposed ion channel panel. J Pharmacol Toxicol Methods. 2016;

Address for correspondence.

Michelangelo Paci

Tampere University of Technology, BioMediTech Arvo Ylpön katu 34, D 219, FI-33520 Tampere, Finland michelangelo.paci@tut.fi 